\title{
Clinical features and serial changes of Acanthamoeba keratitis: an in vivo confocal microscopy study
}

\author{
Suxia $\mathrm{Li}^{1,2} \cdot$ Jiang $\mathrm{Bian}^{2} \cdot$ Yuting $\mathrm{Wang}^{2} \cdot$ Shuting $\mathrm{Wang}^{2} \cdot$ Xin $\mathrm{Wang}^{2} \cdot$ Weiyun Shi $\mathbb{C}^{1,2}$
}

Received: 1 August 2017 / Revised: 10 December 2018 / Accepted: 2 April 2019 / Published online: 10 July 2019

(c) The Author(s), under exclusive licence to The Royal College of Ophthalmologists 2019

\begin{abstract}
Purpose To observe the clinical features and serial changes of Acanthamoeba keratitis (AK) during medical treatment by using confocal microscopy.

Methods Thirty-seven patients (37 eyes) diagnosed with AK were included in this study. Confocal microscopy was used to observe the morphology, distribution, and density of Acanthamoeba cysts before and after medication. The differences between cysts and inflammatory cells were identified.

Results Acanthamoeba cysts were detected at a rate of $94.6 \%$ (35/37) by repeated confocal microscopic examinations. The cysts consisting of a lowly light-reflective wall and a high-refractive nucleus, showed cluster or chain distribution in the corneal stroma, which was different from inflammatory cells. After medical therapy, the nucleus of cysts or peripheral corneal tissue gradually dissolved to a hollow configuration. Some of the hollow cysts existed for up to 6 months. The quantity of cysts increased after 1-2 weeks of medication in 23 patients $(62.1 \%)$, and then began to decrease in 13 patients (35.1\%) who were responsive to anti-amoebic treatment.

Conclusion Acanthamoeba cysts have many typical clinical features that can be identified by confocal microscopy, which may serve as a valuable tool to guide clinical evaluation and treatment of AK.
\end{abstract}

\section{Introduction}

Acanthamoeba keratitis (AK) is a relatively unusual corneal disease infected by amoeba protozoa and shows complex clinical manifestations. As its clinical diagnosis is sometimes difficult, it is often misdiagnosed and treated as other types of keratitis, such as viral keratitis and fungal keratitis [1]. This leads to a delay in initiating appropriate treatments and will result in poor outcomes. Early and accurate diagnosis is particularly important for the treatment of this intractable eye disease [2].

Definitive diagnosis can be made by confirmation of Acanthamoeba cysts or trophozoites in corneal lesions by

Weiyun Shi

weiyunshi@163.com

1 State Key Laboratory Cultivation Base, Shandong Provincial Key Laboratory of Ophthalmology, Shandong Eye Institute, Shandong First Medical University \& Shandong Academy of Medical Sciences, Qingdao, China

2 Shandong Eye Hospital, Shandong Eye Institute, Shandong First Medical University \& Shandong Academy of Medical Sciences, Jinan, China staining, tissue culturing or pathology. However, these methods are invasive, time-consuming and not always routinely available [3-5]. Because of the low sensitivity of Acanthamoeba cultures, which is reported to be just 54-68\% [3]; the effectiveness of isolating Acanthamoeba in cultures is not so high, and it usually takes 1-2 weeks to obtain the results [6]. To improve the understanding and make a rapid diagnosis of $\mathrm{AK}$, the usefulness of in vivo confocal microscopy has been extensively reported [7-11]. However, it is still somewhat difficult to differentiate Acanthamoeba cysts from other cell populations, especially inflammatory cells. If we know better about the microscopic appearances of amoeba cysts, this rapid and non-invasive tool may be very helpful for early diagnosis of AK and observation of pathogenetic condition of the disease, so as to monitor disease progression and guide treatment. This study introduced the clinical features and serial changes of AK on confocal microscopy.

\section{Patients and methods}

This retrospective, noncomparative case series study was approved by the Institutional Review Board of Shandong 
Eye Hospital. Thirty-seven patients (37 eyes) hospitalised for AK at our institution from December 2007 to July 2015 were included. They were 19 males and 18 females, with a mean age of $48.8 \pm 11.1$ years (range, 18-76 years), including 25 farmers, 3 teachers, 3 workers, 2 civil servants, 1 nurse and three unemployed persons. The mean disease duration was $38 \pm 25$ days (range, 7 days -4 months).

The morphology, position and invasive depth of corneal lesions and hypopyon observed by slit-lamp microscopy were recorded. No endophthalmitis was found in any eye on ultrasonic inspection.

\section{Laser confocal microscopy examination and serial observation}

Laser confocal microscopy (HRT3-RCM; Heidelberg Engineering, Heidelberg, Germany) was performed with the Zeiss built-in camera in patients with corneal infection, and in those highly suspected for AK. The detection was repeated three times, once a day by different technicians, to improve the positivity rate of Acanthamoeba cysts. Once diagnosed as $\mathrm{AK}$, the superior, inferior, temporal, nasal and central parts of the corneal infiltration were scanned [12]. The size, morphology, distribution and density of the cysts were evaluated and differentiated from the inflammatory cells by a masked observer. The cysts were calculated using the analysis software of the instrument, and the numbers from five randomly chosen images in each position were averaged. After initiation of treatment, the patients were examined at 1 week, 2 weeks, 1 month, 2 months and 6 months, respectively. Changes of cysts before and after medication were compared.

\section{Corneal scraping and culture}

Necrotic and infiltrated tissues of the corneal ulcer were scraped by experienced doctors under a microscope for $10 \%$ potassium hydroxide wet mount preparations. Corneal samples were cultured in the medium with Escherichia coli, and the temperature of the wet box was controlled at $35^{\circ} \mathrm{C}$. It was regarded as a negative result if there was no Acanthamoeba growth within 14 days.

\section{Pathologic examination}

Corneal tissues were harvested from the patients receiving keratoplasty. The pathological sections stained with hematoxylin eosin were observed for amoeba cysts.

\section{Medical therapy}

Anti-amoebic therapy was initiated once AK was diagnosed. Chlorhexidine eyedrops $(0.02 \%)$ were administered every half an hour; metronidazole eyedrops $(0.5 \%)$ were given per hour; ofloxacin and pranopulin eyedrops were given every $6 \mathrm{~h}$ per day. Intravenous injection of metronidazole was performed once daily. Cauterisation with $(0.2 \%)$ chlorhexidine solution was applied, if necessary. The frequency of medical administration was adjusted under guidance of confocal microscopy imaging. Keratoplasty and even eye evisceration were performed for patients with advanced AK incontrollable with the drug treatment.

\section{Results}

\section{Diagnosis of AK}

Among the 37 patients with $\mathrm{AK}$, the positive rate of corneal scraping staining and culture was $80.7 \%(25 / 31)$ and $76.7 \%$ (23/30), respectively. Acanthamoeba cysts were detectable in 17 pathological specimens $(89.5 \%)$ extracted from 19 patients and in 33 (94.6\%) of 35 patients by confocal microscopy. There were 23 patients $(62.2 \%)$ showing positivity on both confocal microscopy and culture, 17 patients (45.9\%) showing positivity on both confocal microscopy and pathology, and 14 patients (37.8\%) showing positivity on all the three tests. At the early stage of AK, oedematous epitheliopathy of the central cornea was observed by slit-lamp microscopy. The eyes with $\mathrm{AK}$ at the mid-late stage (16 eyes, $44 \%$ ) were characterised by ring infiltrates, with individual differences in size. However, some patients demonstrated diffusive infiltration. Five patients $(13.5 \%)$ had hypopyon. Thirteen patients were cured completely by medical therapy. Sixteen patients received lamellar keratoplasty (6 patients) or penetrating keratoplasty (10 patients). Three patients received evisceration. Five patients were lost for follow-up.

\section{Identification of Acanthamoeba cysts}

Cysts are usually round, consisting of an outer capsular wall and an inner nucleus. The images obtained from in vivo confocal microscopy presented a highly reflective nucleus surrounded by a low-refractile cyst wall, which had a clear boundary and a dark ring outside (Fig. 1a-c). All cysts were $5-20 \mu \mathrm{m}$ in diameter, and mostly $10-15 \mu \mathrm{m}$. The major central structures were regular rounds with uniform reflection (Fig. 1a, b). Irregular shapes, like a triangle (Fig. 1d), an asterisk (Fig. 1e) or a hollow ring (Fig. 1f), were also observed.

Twenty-six eyes (70.3\%) showed a chain-like arrangement of 3-6 cysts. This specific arrangement was consistent with the results of culture and pathology (Fig. 2a-f). More typically, a mass of cysts (3-15) arranged in an arc along the corneal stroma fibres. The adjacent cysts were parallel 
Fig. 1 Laser confocal microscopic images of amoeba cysts. The cyst shows a highly reflective nucleus surrounded by a low-refractile ring-like wall $(\mathbf{a}-\mathbf{c})$. The central structure is regular and round with uniform reflection $(\mathbf{a}, \mathbf{b})$, or is shaped like a triangle (d), an asterisk (e) or a hollow ring (f)
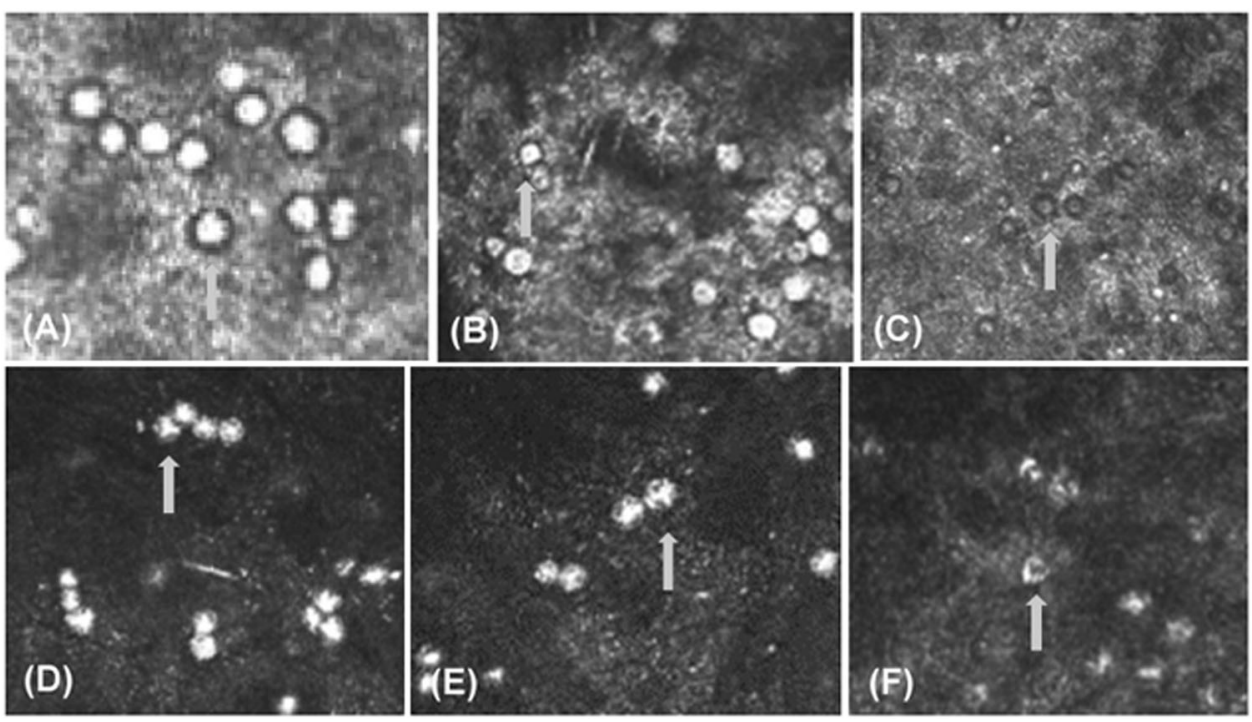

and equidistant from each other. Even the dense cysts with a close distance had tiny space between each other and did not overlap (Fig. 2g, h).

\section{Distinguishing Acanthamoeba cysts from inflammatory cells}

Most inflammatory cells were of an irregularly oval shape. The structure of inflammatory cells, such as neutrophils, was featured with a lobate nucleus, which had a lower light-reflection than cysts, and did not have an external wall. The arrangement of inflammatory cells, under the same field of view, was asymmetric. The cells overlapped and squeezed with each other and distributed in different planes of the corneal stroma (Fig. 2i, j). The differences between amoeba cysts and inflammatory cells are presented in Table 1.

\section{Duration of the treatment}

In vivo confocal microscopy showed that the nuclei in cysts gradually dissolved to hollow structures (Fig. 3a, b). Corneal tissues were dissolved and denatured, showing a low reflective area like a black hole around cysts (Fig. 3c, d).

The quantity of cysts increased after 1-2 weeks of medical therapy in 23 eyes $(62.1 \%)$, and then began to decrease with further treatment. That is, the quantitative peak of cysts appeared at $\sim 2$ weeks after treatment, when confocal microscopy showed clear and typical cysts. In the 13 patients $(35.1 \%)$ who were sensitive to anti-amoebic drugs, a remarkable reduction in the number of cysts was found after 2 months of treatment (Fig. $4 \mathrm{a}-\mathrm{h}$ and o). However, the cysts were monitored to last 6-10 months in a few patients, even though the ulcer was healed. In the other patients who did not respond well to drug treatment, the number of cysts kept at a high level for months and the density of the cyst core showed no significant decrease and even increased in some eyes (Fig. $4 \mathrm{i}-\mathrm{n}$ and o).

\section{Monitoring and treatment guidance by confocal microscopy}

Confocal microscopy was performed after the corneal ulcer healed up with medication in 13 patients. Suspected amoeba cysts were still present, so drug treatment was continued, until the cysts disappeared or just the hollow structures remained. According to the lesion depth and the cyst scope scanned by confocal microscopy, the patients who failed in medical therapy were considered for lamellar keratoplasty or penetrating keratoplasty and even evisceration due to the involvement of the sclera.

\section{Discussion}

AK is an infectious disease caused by amoebas, which survive in air, soil, lake water, tap water and dust as trophozoites or cysts [13]. Trophozoites are active and reproductive in suitable circumstances, while cysts are transformed from trophozoites and can resist severe environment. The static form, known as amoeba cysts, has a double wall for resisting outer adversity [14]. The low incidence and lack of related knowledge pose a diagnostic dilemma for AK. Typical but non-specific clinical manifestations of $\mathrm{AK}$ include early severe pain, infiltration in or under the corneal epithelium, corneal ring infiltrates and hypopyon [9, 15-17]. At the early stage of AK, central corneal oedema is very similar to that of viral keratitis. The misdiagnosis rate is high for $\mathrm{AK}$ at the late or advanced phase because the corneal ulcer of AK often 

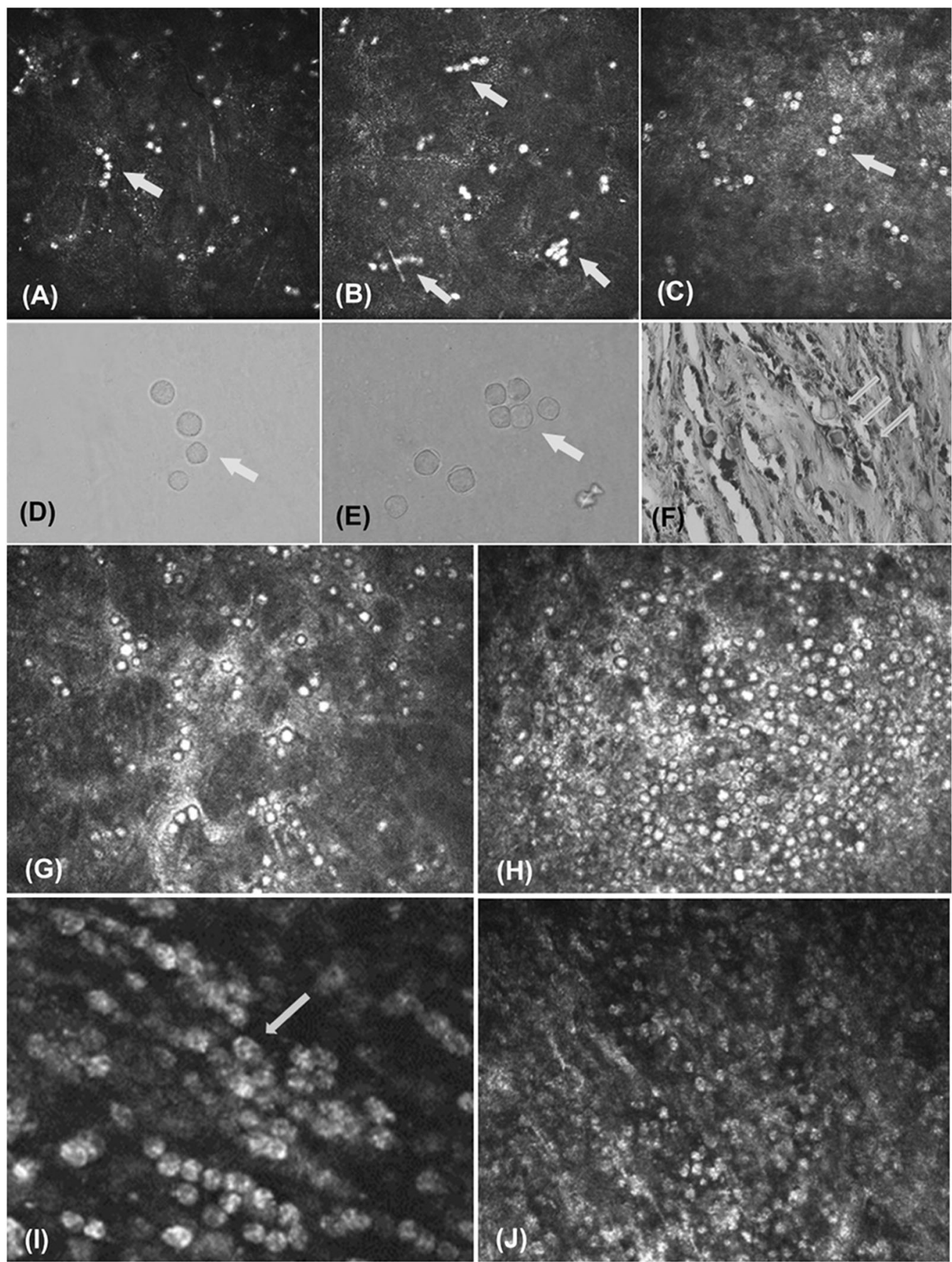

Fig. 2 The Acanthamoeba cysts distribute like chains with 3-6 cysts $(\mathbf{a}-\mathbf{c})$. The culture and pathology results show the same distribution features $(\mathbf{d}-\mathbf{f})$. Even if there are a large number of cysts, they do not overlap and have a tiny space between each other $(\mathbf{g}, \mathbf{h})$. Most

shows a similar shape with fungal keratitis [18-20]. Once patients with $\mathrm{AK}$ are misdiagnosed as any other disease, especially viral keratitis, and given glucocorticoids, the corneal ulcer could be aggravated, uncontrollable and even violate the entire eye globe. Therefore, early and accurate diagnosis is crucial for therapy and prognosis of patients affected with AK. inflammatory cells are of an irregularly oval shape with a lobate nucleus and do not have an external wall (i). The arrangement of inflammatory cells is asymmetric, overlapped and squeezed with each other, and distributed in different planes of the corneal stroma $(\mathbf{j})$

The main auxiliary examinations for AK include corneal scraping, amoeba culture and pathology. Although corneal scraping is an effective and fast method for AK diagnosis, the positive rate may be relatively low in the early stage of the disease. Because of the low sensitivity of Acanthamoeba cultures, it usually takes at least 1 week to obtain the outcomes [6], and the positive rate was reported to be just 
Table 1 Differences between Acanthamoeba cysts and inflammatory cells

\begin{tabular}{lll}
\hline & Acanthamoeba cysts & Inflammatory cells \\
\hline Shape & Round & Oval or irregular \\
Wall & Low refractile and circular area & N/A \\
Nucleus & High-light reflection and regular rounds, or & Lower light reflection, eccentricity, \\
& irregular shape like Chinese characters or & uneven density and lobate nuleus \\
& hollow rings & Irregular \\
Arrangement & Chain-like and regular & Un-equidistant, overlapped and \\
Spacing & Equidistant and non-overlapped & squeezed \\
\hline
\end{tabular}

N/A not available
Fig. 3 Confocal microscopic images showing Acanthamoeba cyst changes after drug treatment. The nuclei in cysts gradually dissolve to hollow structures $(\mathbf{a}, \mathbf{b})$. Some cysts dissolve the corneal tissue around them, and form a black hole $(\mathbf{c}, \mathbf{d})$
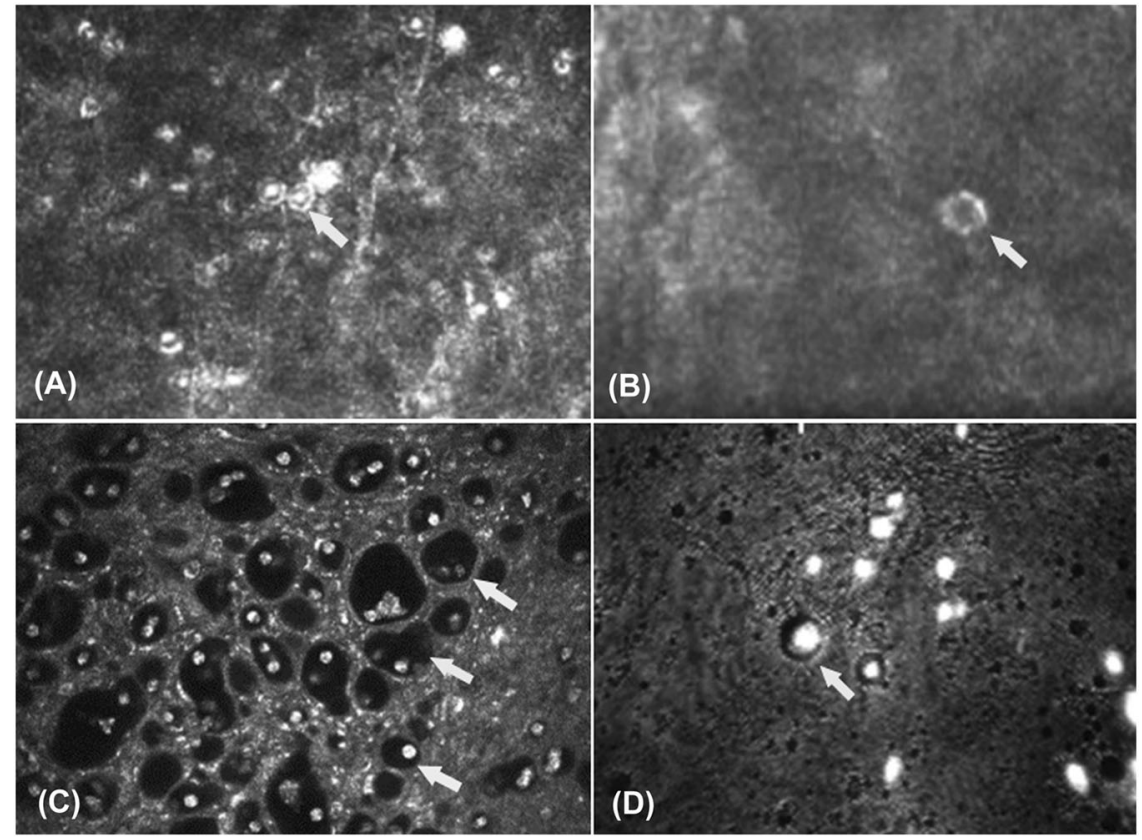

54-68\% [3]. Pathologic examinations need corneal tissues resected from patients during keratoplasty; hence, it is impossible for early diagnosis. Polymerase chain reaction was reported to detect amoeba pathogens quickly and efficiently [21-23], but this technique is often limited in clinical practice due to the requirement for specialised instruments and laboratories.

Since Chew et al. [24] firstly reported the diagnosis of AK using confocal microscopy; this fast, non-invasive, repeatable and intuitive technology has been gradually employed in detecting AK, particularly at the early stage when clinical manifestations are not typical. However, it is not easy to distinguish Acanthamoeba cysts from inflammatory cells due to their similarities. Thus, the positivity rate of detecting $\mathrm{AK}$ by confocal microscopy seems to be low. In this study, the positivity rate by in vivo confocal microscopy was as high as $94.6 \%$, surpassing corneal scarping $(80.7 \%)$ and amoebic culture (76.7\%). Knowing the clinical features of cysts on confocal microscopy and detecting repeatedly can help to improve the detection rate.

Just like fungal hyphae observed by laser confocal microscopy, amoeba cysts can be recognised clearly with typical morphology and distribution. Winchester et al. [25] demonstrated confocal microscopic images of Acanthamoeba cysts featured with a high-reflective, double-walled, round structure, $10-30 \mu \mathrm{m}$ in diameter, in ten cases. Some researchers introduced the crucial role of in vivo confocal microscopy in early diagnosis for AK. In our series, we observed the patients with AK by confocal microscopy and summarised the features of Acanthamoeba cysts. In brief, each cyst consisted of a lowly light-reflective wall and a high-refractile nucleus, and connected with each other like chains in the same plane of corneal stroma. Cysts showing a cluster or chain-like arrangement and locating in the same plane of corneal stroma might be motor direction related, reminding us of enlarging the resection area of the cornea to reduce the recurrence of $\mathrm{AK}$. Whether this distribution 

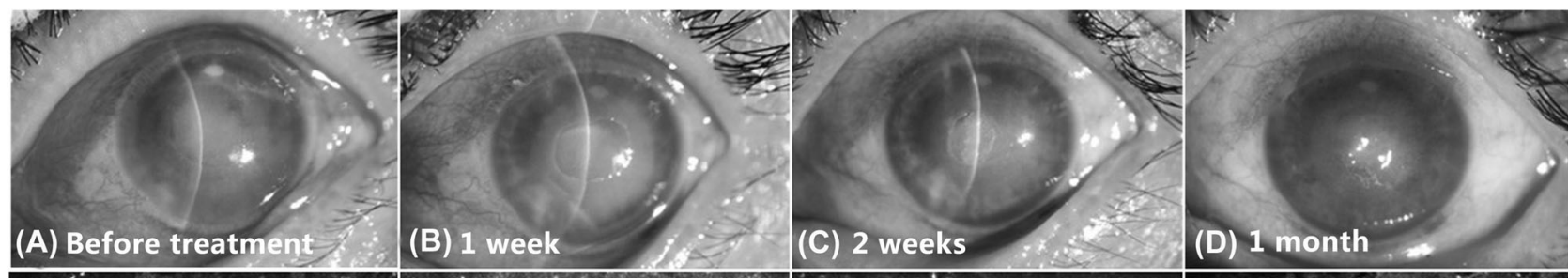

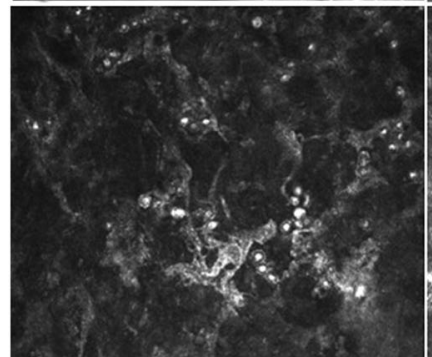

(E), Before treatment
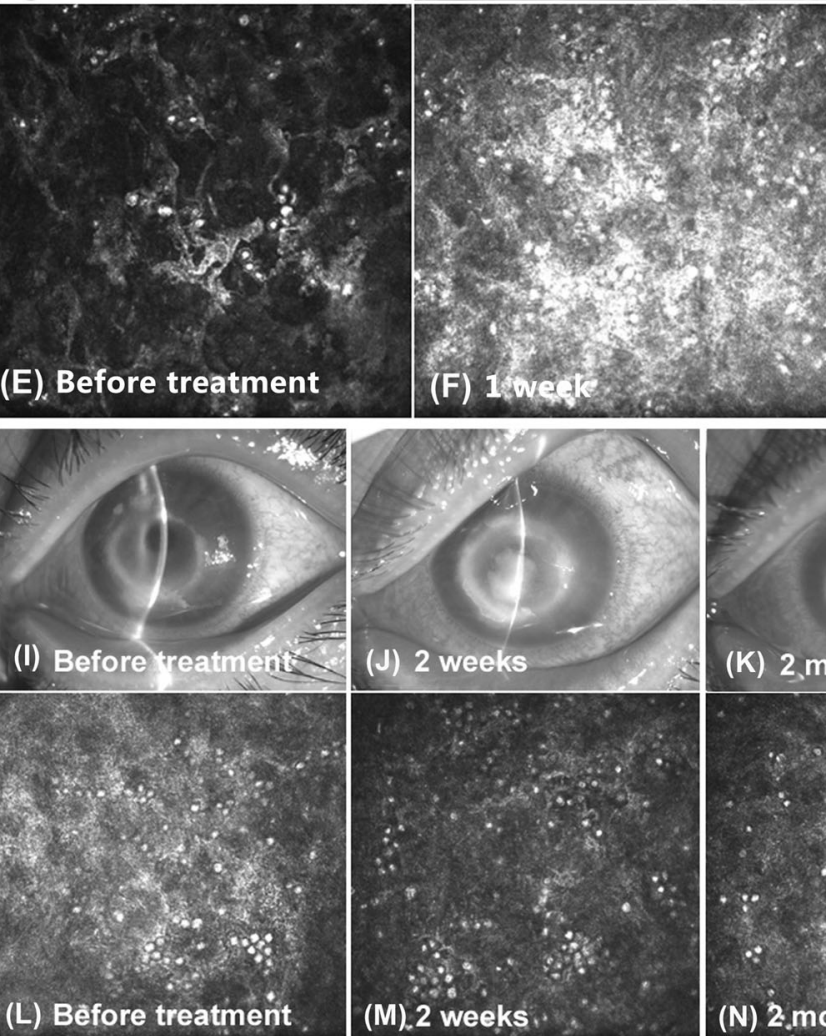
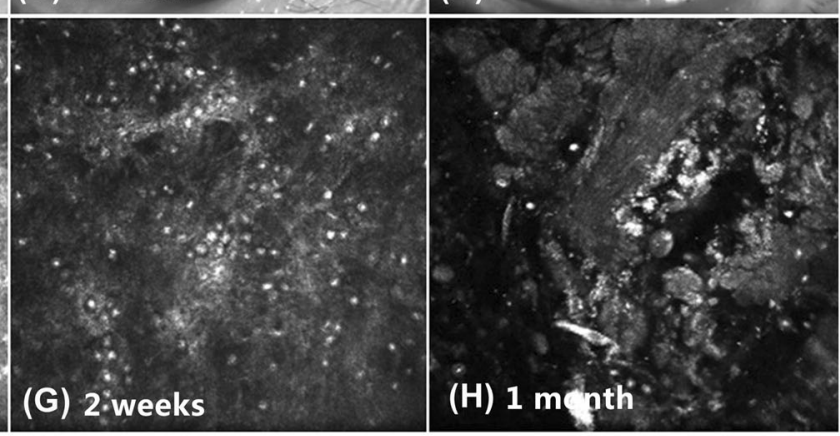
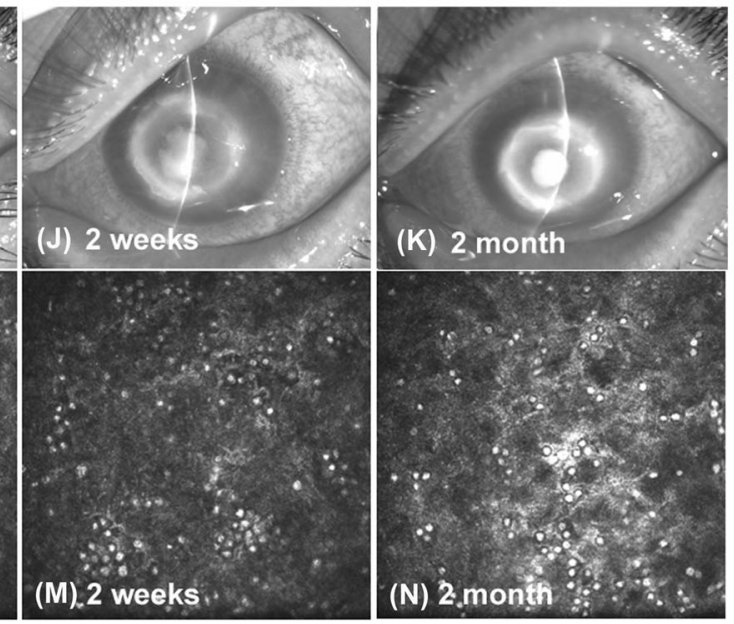

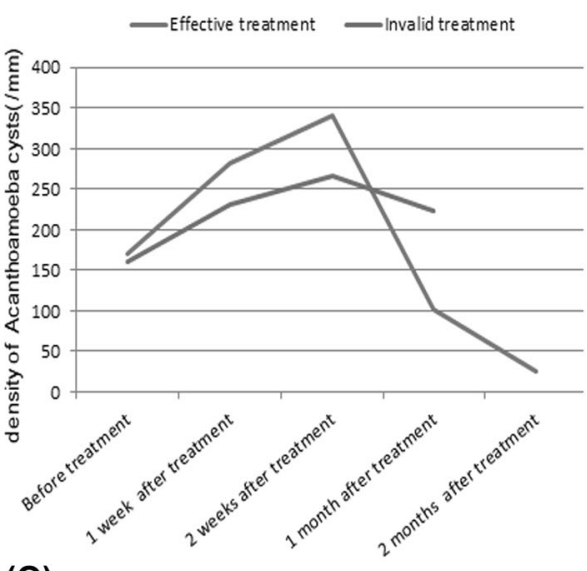

(0)
Fig. 4 Therapeutic outcomes of Acanthamoeba keratitis (AK) in patients who are sensitive to medication. The number of cysts increases after 2-week treatment and begins to decrease with the further medical therapy $(\mathbf{a}-\mathbf{h})$. In patients who respond poorly to drugs,

pattern is related to the orientation of corneal nerves deserves further investigations. The images of Acanthamoeba cysts and inflammatory cells shown by confocal microscopy can be easily confused, so we made detailed comparisons of these similarities and differences for better identification. The morphology and number of cysts changed with the medical therapy. After drug treatments, nuclei of cysts or peripheral corneal tissue gradually dissolved to hollow configurations. The number of cysts firstly increased and then decreased after effective treatment. We speculated these outcomes were due to strong resistance of cysts to anti-amoebic drugs, and other physical and chemical factors [26]. In other patients who poorly responded to drug treatment, cysts kept increasing in number or decreased insignificantly during the therapy. The features of Acanthamoeba cysts we described above helped guide the diagnosis and treatment of AK. the cyst quantity increases after treatment and keeps at a high level for months (i-n). Line graph shows the quantity change of Acanthamoeba cysts $(\mathbf{o})$

The amoeba trophozoites can be transformed into cysts under adverse surroundings. Usually the trophozoites could not be detected by confocal microscopy, while cysts can. On our observation, most of the patients showed an improvement in the quantity of cysts after medication, including those who were sensitive to the drug treatment. This may be because some of the trophozoites were transformed to cysts due to medical therapy; and thus easily detected by confocal microscopy, and 2-weeks appears to be the peak time for them to transform. The results of dynamic observation of amoeba cysts can guide the diagnosis and management of AK. Early after the anti-amoebic medication, more amoeba cysts would accumulate. When a good therapeutic effect is achieved, the number of cysts usually decreases. If there is no significant decrease, this indicates that amoeba cysts are not sensitive to the drug therapy, when keratoplasty should be considered. 
Moreover, the depth of infiltration determined by confocal microscopy is very useful in selecting penetrating keratoplasty or lamellar keratoplasty.

In conclusion, Acanthamoeba cysts show typical morphology and chains-like arrangement on confocal microscopy. The change in the number of cysts has its regularity after medication. The diagnosis rate of AK by laser in vivo confocal microscopy can be high, with better understanding of the clinical features of Acanthamoeba cysts. Repeated examinations are still needed to increase sensitivity.

\section{Summary}

\section{What was known before}

- In vivo confocal microscopy has been used in detection of Acanthamoeba cysts and diagnosis of Acanthamoeba keratitis.

\section{What this study adds}

- Clinical features of Acanthamoeba cysts in more details are observed and described.

- It is the first time to describe the changes of Acanthamoeba cysts after medical treatment.

- Differentiation of Acanthamoeba cysts from inflammatory cells is introduced.

Acknowledgements We thank Ping Lin, MTI for her linguistic and editorial assistance.

Funding This study was supported by the National Natural Science Foundation of China (81500702, 81530027), the Shandong Provincial Natural Science Foundation (2016GSF201216), the Taishan Scholar Program Phase II (tspd20150215) and the Innovation Project of Shandong Academy of Medical Sciences.

\section{Compliance with ethical standards}

Conflict of interest The authors declare that they have no conflict of interest.

Publisher's note: Springer Nature remains neutral with regard to jurisdictional claims in published maps and institutional affiliations.

\section{References}

1. Hammersmith KM. Diagnosis and management of Acanthamoeba keratitis. Curr Opin Ophthalmol. 2006;17:327-31.

2. Thomas PA, Geraldine P. Infectious keratitis. Curr Opin Infect Dis. 2007;20:129-41.
3. Tu EY, Joslin CE, Sugar J, Booton GC, Shoff ME, Fuerst PA. The relative value of confocal microscopy and superficial corneal scrapings in the diagnosis of Acanthamoeba keratitis. Cornea. 2008;27:764-72.

4. Bacon AS, Frazer DG, Dart JK, Matheson M, Ficker LA, Wright P. A review of 72 consecutive cases of Acanthamoeba keratitis, 1984-1992. Eye. 1993;7:719-25.

5. Parmar DN, Awwad ST, Petroll WM, Bowman RW, McCulley JP, Cavanagh HD. Tandem scanning confocal corneal microscopy in the diagnosis of suspected Acanthamoeba keratitis. Ophthalmology. 2006;113:538-47.

6. Labbé A, Khammari C, Dupas B, Gabison E, Brasnu E, Labetoulle $\mathrm{M}$, et al. Contribution of in vivo confocal microscopy to the diagnosis and management of infectious keratitis. Ocul Surf. 2009;7:41-52.

7. Kaufman SC, Musch DC, Belin MW, et al. Confocal microscopy: a report by the American Academy of Ophthalmology. Ophthalmology. 2004;111:396-406.

8. Mathers WD, Nelson SE, Lane JL, Wilson ME, Allen RC, Folberg R. Confirmation of confocal microscopy diagnosis of Acanthamoeba keratitis using polymerase chain reaction analysis. Arch Ophthalmol. 2000;118:178-83.

9. Kobayashi A, Yokogawa H, Yamazaki N, Ishibashi Y, Oikawa Y, Tokoro M, et al. In vivo laser confocal microscopy findings of radial keratoneuritis in patients with early stage Acanthamoeba keratitis. Ophthalmology. 2013;120:1348-53.

10. Zhang X, Sun X, Jiang C, Wang Z, Zhang Y, Deng S, et al. A new in vivo confocal microscopy prognostic factor in Acanthamoeba keratitis. J Fr Ophtalmol. 2014;37:130-7.

11. Füst Á, Tóth J, Simon G, Imre L, Nagy ZZ. Specificity of in vivo confocal cornea microscopy in Acanthamoeba keratitis. Eur J Ophthalmol. 2017;27:10-15.

12. Liu M, Gao H, Wang T, Wang S, Li S, Shi W. An essential role for dendritic cells in vernal keratoconjunctivitis: analysis by laser scanning confocal microscopy. Clin Exp Allergy. 2014; 44:362-70.

13. Khan NA. Acanthamoeba: biology and increasing importance in human health. FEMS Microbiol Rev. 2006;30:564-95.

14. Stern GA. Contact lens associated bacterial keratitis: past, present, and future. CLAO J. 1998;24:52-6.

15. Chin J, Young AL, Hui M, Jhanji V. Acanthamoeba keratitis: 10Year study at a tertiary eye care center in Hong Kong. Cont Lens Anterior Eye. 2015;38:99-103.

16. Wang Y, Feng X, Jiang L. Current advances in diagnostic methods of Acanthamoeba keratitis. Chin Med J (Engl). 2014;127:3165-70.

17. Iovieno A, Gore DM, Carnt N, Dart JK. Acanthamoeba sclerokeratitis: epidemiology, clinical features, and treatment outcomes. Ophthalmology. 2014;121:2340-7.

18. Clarke B, Sinha A, Parmar DN, Sykakis E. Advances in the diagnosis and treatment of acanthamoeba keratitis. J Ophthalmol. 2012;2012:484892.

19. Wilhelmus KR, Jones DB, Matoba AY, Hamill MB, Pflugfelder SC, Weikert MP. Bilateral acanthamoeba keratitis. Am J Ophthalmol. 2008;145:193-7.

20. da Rocha-Azevedo B, Tanowitz HB, Marciano-Cabral F. Diagnosis of infections caused by pathogenic free-living amoebae. Inter Perspect Infect Dis. 2009;2009:251406.

21. Ikeda Y, Miyazaki D, Yakura K, Kawaguchi A, Ishikura R, Inoue $\mathrm{Y}$, et al. Assessment of real-time polymerase chain reaction detection of Acanthamoeba and prognosis determinants of Acanthamoeba keratitis. Ophthalmology. 2012;119:1111-9.

22. Khairnar K, Tamber GS, Ralevski F, Pillai DR. Comparison of molecular diagnostic methods for the detection of Acanthamoeba spp. from clinical specimens submitted for keratitis. Diagn Microbiol Infect Dis. 2011;70:499-506. 
23. Laummaunwai P, Ruangjirachuporn W, Boonmars T. A simple PCR condition for detection of a single cyst of Acanthamoeba species. Parasitol Res. 2012;110:1569-72.

24. Chew SJ, Feuerman RW, Assouline M, Kaufman HE, Barron BA, Hill JM. Early diagnosis of infectious keratitis with in vivo real time confocal microscopy. CLAO J. 1992;18:197-201.
25. Winchester K, Mathers WD, Sutphin JE, Daley TE. Diagnosis of Acanthamoeba keratitis in vivo with confocal microscopy. Cornea. 1995;14:10-7.

26. Aksozek A, McClellan K, Howard K, Niederkorn JY, Alizadeh H. Resistance of Acanthamoeba castellanii cysts to physical, chemical, and radiological conditions. J Parasitol. 2002;88:621-3. 